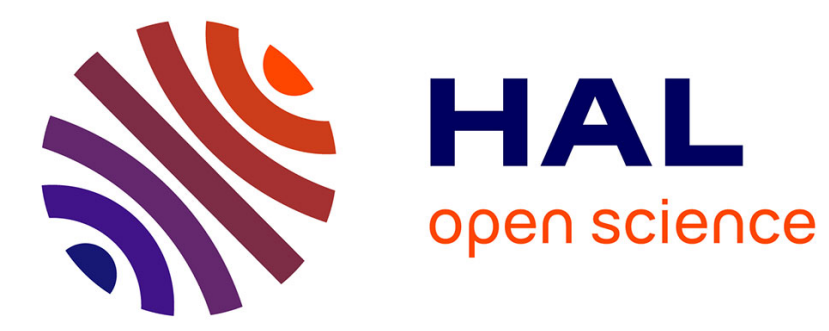

\title{
Optimally controlling the internal dynamics of a randomly oriented ensemble of molecules
}

\author{
Gabriel Turinici, Herschel Rabitz
}

\section{To cite this version:}

Gabriel Turinici, Herschel Rabitz. Optimally controlling the internal dynamics of a randomly oriented ensemble of molecules. Physical Review A : Atomic, molecular, and optical physics [1990-2015], 2004, 70 (6), pp.063412. 10.1103/PhysRevA.70.063412 . hal-00798257

\section{HAL Id: hal-00798257 \\ https://hal.science/hal-00798257}

Submitted on 8 Mar 2013

HAL is a multi-disciplinary open access archive for the deposit and dissemination of scientific research documents, whether they are published or not. The documents may come from teaching and research institutions in France or abroad, or from public or private research centers.
L'archive ouverte pluridisciplinaire HAL, est destinée au dépôt et à la diffusion de documents scientifiques de niveau recherche, publiés ou non, émanant des établissements d'enseignement et de recherche français ou étrangers, des laboratoires publics ou privés. 


\title{
Optimally controlling the internal dynamics of a randomly oriented ensemble of molecules
}

\author{
Gabriel Turinici* \\ INRIA Rocquencourt, Domaine de Voluceau, \\ Rocquencourt B.P. 105, 78153 Le Chesnay Cedex, France ${ }^{\dagger}$ \\ Herschel Rabitz \\ Department of Chemistry, Princeton University, Princeton, NJ 08544-1009, USA
}

(Dated: October 6, 2004)

\begin{abstract}
The ultra-fast control of large polyatomic molecules in the gas and condensed phases entails working with a randomly oriented ensemble. During the short control period, little reorientation may occur especially for cases in the condensed phases. This paper addresses the degree to which all members of the ensemble may be simultaneously controlled with respect to their internal motion by a single laser pulse. It is shown that all members of the ensemble are fully controllable if any one member is. Numerical optimal control simulations also show that excellent quality full ensemble control can be achieved even with reasonable constraints placed on the control fields. Although the full ensemble may be controlled to a high degree, the control mechanism is likely to differ for each ensemble member.
\end{abstract}

PACS numbers: $32.80 . \mathrm{Qk}$

Keywords: quantum control, controllability, bilinear system, orientation

*Electronic address: Gabriel.Turinici@inria.fr

${ }^{\dagger}$ CERMICS-ENPC, Champs sur Marne, 77455 Marne la Vallée Cedex, France

${ }_{\ddagger}$ Electronic address: hrabitz@princeton.edu 


\section{INTRODUCTION}

There is much interest in the control of molecular dynamics phenomena including chemically reactive processes in large polyatomic molecules [1]. Recent experiments [2-8] have demonstrated the feasibility of meeting these goals especially through the use of closed loop adaptive techniques [9] operating directly in the laboratory. Successful experiments range from the manipulation of diatomic molecules out through large polyatomic molecules and even protein complexes, with studies in the gas and liquid phase environments. The shaped laser pulses involved can operate over a time-scale of approximately 1ps or even less. Theoretical $[10,11]$ and experimental [12] work has shown that molecular re-orientation can take place for small molecules in the gas phase, especially after the pulses are over due to rotational coherences that are generated. However, large polyatomic molecules are expected to undergo little re-orientation during the pulse period especially in the confined environment of condensed phases. The adaptive control experiments will seek a single optimal control field that manipulates the internal dynamics of all members of the randomly oriented molecular ensemble as best as possible. This paper addresses whether the control field must strike a compromise in trying to manipulate the full ensemble of randomly oriented molecules.

Although each member of the molecular ensemble is identical, in the presence of the control electric field vector this symmetry is broken, as the field will interact in a distinct way with each molecule that is oriented differently. Thus, the problem of controlling the internal dynamics of the entire ensemble with a single field is very similar to the problem of controlling a collection of physically distinct molecules. The latter topic has been the subject of controllability and optimal control analyses[13, 14]. In the present work, an ensemble of randomly oriented identical molecules is first subjected to a controllability analysis in Section II. This analysis shows that if any member of the ensemble is controllable then the entire ensemble should be controllable. This very strong positive result is rather counterintuitive and it arises as a result of the nonlinearity of quantum control.

The practical reality of fully controlling an ensemble of randomly oriented molecules is explored in Section III with optimal control simulations on a model system. The formal theoretical conclusions on controllability in Section II are confirmed to an excellent degree in the simulations even while working with limitations placed on the controls. However, it is observed that different members of the ensemble evolve to each have a distinct control 
mechanism while still achieving similar yields in the target state. Finally, Section IV presents a brief summary of the findings and comments on their significance.

\section{ORIENTATIONAL ENSEMBLE CONTROL}

\section{A. The ensemble quality control index}

Consider an ensemble of molecules exposed to an ultrafast laser control electric field $\varepsilon(t)$ such that little rotational motion of any molecule occurs during the laser pulse. This condition can depend on the pulse length, field strength and the sample medium; satisfaction of this condition is the fundamental assumption in this work. The assumption will be increasingly valid for larger molecules and especially in condensed phase environments. The goal here is not to explicitly model such complex circumstances, but rather to understand what may happen to the ensemble under optimal control in the laboratory. The unperturbed Hamiltonian $H_{0}$ will now just depend on the internal molecular degrees of freedom and the control goal is to optimally manipulate the internal dynamics of the molecules. Each member of the ensemble is characterized by the angle $\theta$ between the dipole operator $\mu$ and the field vector $\varepsilon(t)$ producing the Hamiltonian $H=H_{0}-\cos (\theta) \mu \varepsilon(t)$. The molecules evolve under the Schrödinger equation

$$
\begin{aligned}
& i \hbar \frac{\partial}{\partial t}|\Psi(t, \theta)\rangle=\left(H_{0}-\cos (\theta) \mu \varepsilon(t)\right)|\Psi(t, \theta)\rangle \\
& \Psi\left(t_{0}, \theta\right)=\Psi^{0}(\theta) .
\end{aligned}
$$

A more general formulation would employ the density matrix with the Liouville equation

$$
\begin{aligned}
& i \hbar \frac{\partial}{\partial t} \rho(t, \theta)=\left[H_{0}-\cos (\theta) \mu \varepsilon(t), \rho(t, \theta)\right] \\
& \rho\left(t_{0}, \theta\right)=\rho^{0}(\theta) .
\end{aligned}
$$

The two formulations can be expressed conveniently using the time evolution operator $U\left(t, t_{0}\right)_{\theta}$ given by

$$
\begin{aligned}
& i \hbar \frac{\partial}{\partial t} U\left(t, t_{0}\right)_{\theta}=\left(H_{0}-\cos (\theta) \mu \varepsilon(t)\right) U\left(t, t_{0}\right)_{\theta} \\
& U\left(t_{0}, t_{0}\right)_{\theta}=I .
\end{aligned}
$$


as $\Psi(t, \theta)=U\left(t, t_{0}\right)_{\theta} \Psi\left(t_{0}, \theta\right)$ and $\rho(t, \theta)=U\left(t, t_{0}\right)_{\theta} \rho\left(t_{0}, \theta\right) U\left(t, t_{0}\right)_{\theta}^{\dagger}$. The molecules are assumed to be described in terms of a discrete basis of dimension $N$.

An important question is whether a single laser field $\varepsilon(t)$ is able to simultaneously control the full internal dynamics of the ensemble of differently oriented molecules. Of course, one preliminary requirement is that the laser field be capable of controlling at least one individual member at some angle $\bar{\theta}$. This requirement has been addressed in different contexts [1519] and the criterion can be adapted to the present situation involving the $N$-dimensional matrices of the operators $H_{0}$ and $\cos (\theta) \mu$ with $\theta$ being a parameter. Suppose that for one value $\bar{\theta}$ the matrices $H_{0}$ and $\cos (\bar{\theta}) \mu$ span the Lie algebra $u(N)$ (or $s u(N)$ if both matrices are traceless). Then this selected set of molecules is controllable in the sense that from any initial state $\Psi_{i}$ (of unit norm $\left\|\Psi_{i}\right\|_{L^{2}}=1$ ) any other state $\Psi_{f}$ (of unit norm $\left\|\Psi_{f}\right\|_{L^{2}}=1$ ) can be attained with some laser field $\varepsilon(t)$. The same conclusion holds for the density matrix representation, provided that the target density matrix $\rho_{f}$ fulfills the compatibility requirement that $\rho_{f}=U \rho_{i} U^{\dagger}$ where $U$ is a unitary matrix.

It is interesting to note that this latter criterion does not depend on the particular value of the angle $\bar{\theta}$ : if individual controllability holds for one value $\bar{\theta}$ then it will hold for any other angle $\theta^{\prime} \neq \pm \pi / 2$. This can be seen by a simple scaling argument: let $\varepsilon(t)$ be the control that achieves the target for $\bar{\theta}$; then $\varepsilon^{\prime}(t)=\cos \left(\theta^{\prime}\right) * \varepsilon(t) / \cos (\bar{\theta})$ will drive molecules oriented with angle $\theta^{\prime}$. Thus, if one member of the ensemble is individually controllable, then any other member is as well (provided that $\cos \left(\theta^{\prime}\right) \neq 0$ ). However this observation does not yet permit drawing a conclusion about the controllability of the full ensemble with the same laser field. Above, the laser field needed to be rescaled in order to steer an ensemble member at a different orientation. In contrast, here we seek to assess whether a single laser field exists that can simultaneously control the full ensemble of differently oriented molecules.

For simplicity the system will be expressed in the wave function formulation but all that follows can be adapted to the density matrix. In the case of all the molecules oriented at angle $\theta_{0}$, the control objective at time $T$ can be formulated as maximizing $\left|\left\langle\Psi\left(T, \theta=\theta_{0}\right), \Psi_{f}\right\rangle\right|^{2}$. Here $\left|\Psi_{f}\right\rangle$ is the final target state (e.g. a particular molecular excitation). Similarly, optimal control over an ensemble of molecules with orientation distribution $\eta(\theta)$ can be expressed as maximizing the cost functional $\int_{0}^{\pi}\left|\left\langle\Psi(T, \theta), \Psi_{f}(\theta)\right\rangle\right|^{2} \eta(\theta) d \theta$ where we consider a general dependence of the target state $\left|\Psi_{f}(\theta)\right\rangle$ upon the orientation parameter $\theta$. This includes the important case when the target is the same for all molecules $\left|\Psi_{f}(\theta)\right\rangle=\left|\Psi_{f}\right\rangle$. Addi- 
tionally, the target may be an orientational subset of the ensemble (e.g. those molecules oriented along the field at $\theta=0$ ), provided that the laboratory feedback signal for optimization is sensitive to molecular orientation. A similar treatment also follows for extremizing $\langle\Psi(T, \theta)|O(\theta)| \Psi(T, \theta)\rangle$ where $O(\theta)$ is a Hermitian objective operator, as establishing controllability for $|\Psi(T, \theta)\rangle, \forall \theta$ implies that the expectation value of an arbitrary $O(\theta)$ may also be controlled. In the remainder of the paper the common case of a three dimensional uniform distribution will be considered with $\eta(\theta)=\sin (\theta)$ arising from the polar volume element. In addition the cost functional is normalized so that a value of zero means no control and a value of 1 means perfect control for the full ensemble. We obtain the following quality index $Q(\varepsilon)$ which depends on the control field $\varepsilon$ :

$$
\begin{aligned}
& Q(\varepsilon)=\frac{\int_{0}^{\pi}\left|\left\langle\Psi(T, \theta), \Psi_{f}(\theta)\right\rangle\right|^{2} \sin (\theta) d \theta}{\int_{0}^{\pi} \sin (\theta) d \theta}=\frac{1}{2} \int_{0}^{\pi}\left|\left\langle\Psi(T, \theta), \Psi_{f}(\theta)\right\rangle\right|^{2} \sin (\theta) d \theta \\
& =\frac{1}{2} \int_{-1}^{1}\left|\left\langle\Psi(T, x), \Psi_{f}(x)\right\rangle\right|^{2} d x
\end{aligned}
$$

where $x=\cos (\theta)$. The integral should be approximated well by a sum having modest sampling over the angular variable $x$, because the integrand is expected to only slowly vary as a function of $x$. Thus, we have for $x_{k}=\cos \left(\theta_{k}\right), k=1, \ldots, M$ and $s_{k}=x_{k}-x_{k-1}$

$$
Q(\varepsilon)=\frac{1}{2} \sum_{k=1}^{M}\left|\left\langle\Psi\left(T, x_{k}\right), \Psi_{f}\left(x_{k}\right)\right\rangle\right|^{2} s_{k} .
$$

The simulations will report the quality index in percent as $100 \times Q(\varepsilon)$. The physical goal is to maximize the quality control index $Q(\varepsilon)$ over the field. The first question to consider is whether a collection of $M$ identical molecules with different spatial orientations is in principle fully controllable and this matter is addressed in the Section II B below.

\section{B. Controllability analysis}

The eigenvalues of the internal Hamiltonian $H_{0}$ are denoted by $\lambda_{a}, a=1, \ldots, N$ with the transition frequencies $\omega_{a b}=\lambda_{a}-\lambda_{b}$. It is assumed that a basis has been selected such that $H_{0}$ is diagonal. A graph associated with the system can be constructed [15] by drawing an edge between any two states coupled by the dipole $\mu$ : $G=(V, E), V=\{1, \ldots, N\}$, $E=\left\{(i, j) ; \mu_{i j} \neq 0\right\}$. A molecule of orientation angle $\bar{x}=\cos (\bar{\theta})$ is controllable [19] if the operators $H_{0}$ and $\bar{x} \mu$ span the Lie algebra $u(N)$ (or $s u(N)$ if both matrices are traceless). The collection of molecules with orientations $x_{1}, \ldots, x_{M}$ (all nonzero) is wave function controllable 
if from any initial state $\Psi\left(t=0, x_{k}\right)=\Psi_{i}\left(x_{k}\right), 100 \%$ overlap with any final states $\Psi_{f}\left(x_{k}\right)$, $k=1, \ldots, M$ can be attained at some final time $T$ for $\Psi\left(T, x_{k}\right), k=1, \ldots, M$.

The following fundamental controllability theorem is for the full ensemble of molecules:

Theorem. Suppose the Hamiltonian $H_{0}$ describes controllable molecules at one value of the orientation angle $\bar{x}=\cos (\bar{\theta})$ (thus for any $x \neq 0$ ) and that

$$
\omega_{a b} \neq \omega_{a^{\prime} b^{\prime}}, \text { for all }(a, b) \neq\left(a^{\prime}, b^{\prime}\right),
$$

Then, if $\left|x_{1}\right|, \ldots,\left|x_{M}\right|$ are all different (and nonzero) the collection of molecules with orientations $x_{1}, \ldots, x_{M}$ is wave function controllable. If however there exist $k, k^{\prime}$ such that $x_{k}=-x_{k^{\prime}}$ but the graph $G$ has at least one odd length cycle (which includes, for example, the case where all non-diagonal entries of $\mu$ are nonzero), then the same conclusion holds. In all controllable cases the collection of molecules is also density matrix controllable.

The proof of the Theorem is given in Appendix A. Accepting the result, the reader may skip to Section III presenting a numerical illustration of the Theorem's consequences.

Finally, it should still be noted that controllability of a finite-dimensional subsystem, no matter how large the dimension, need not imply controllability of the infinite-dimensional system.

Having proved the result above for the bilinear setting of quantum mechanics, it is interesting to compare with the analogous result in the linear case. Using Kalman's criterion $[20,21]$ it can be seen that controllability of an ensemble of $M$ systems

$$
d x_{k} / d t=A_{k} x_{k}+\varepsilon(t) b_{k}, k=1, \ldots, M
$$

requires, in addition to individual member controllability, that the spectra of the free evolution operators $A_{k}$ be all distinct (see [22] for similar results in infinite dimensional circumstances). This condition is certainly not satisfied in the present context as the internal Hamiltonians $H_{0}$ are all identical. However, due to the bilinear nature of the Schrödinger equation, controllability is still present. One can conclude that full ensemble control of quantum systems is a nonlinear phenomena.

Remark 1. The Theorem is not the only controllability criteria available in this setting. Other approaches can also be considered, that, for example, involve consideration of the eigenvalues of the dipole moment $\mu$. An additional class of results could be obtained, along 
the lines in [17, 23], for settings where hypothesis (6) is not true but the dipole moment takes a simple form (e.g., the representation where the dipole matrix is diagonal, but that of $H_{0}$ is not diagonal).

The assumptions underlying the Theorem are not very restrictive, and are often expected to be valid. In addition, the criteria above is only sufficient and controllability may still be true in other situations not covered by this result. We can thus conclude that at least one field will exist that can control the full ensemble, except at the singular point $x=0$.

\section{ILLUSTRATIVE EXAMPLE}

This Section aims to illustrate the Theorem above with a simple quantum control example. Among issues of practical importance are the total time and the maximal field intensity required to control an ensemble of molecules. Asking the control field to steer the full ensemble of molecules to the target at the same time $T$ is a more demanding task than the traditional consideration of control of a single ensemble member. Intuitively, it is expected that the total time required to achieve control may be larger than for any one ensemble member; in the illustration below $T$ is held fixed. As the external field $\varepsilon(t)$ is multiplied by the orientation parameter $x$, and we expect ensemble members in the neighborhood around $x=0$ to be more difficult to control when constraints are placed on the field intensity.

As a test case consider control of a model describing vibrational excitation of the $\mathrm{OH}$ bond [24]. Other multilevel model systems were also treated, and the same general behavior and conclusions were found for controlling ensembles of randomly oriented molecules. The numerical simulations were performed by initially finding an optimal control field for the case $x=1$. Starting from this solution as an initial guess another optimization was performed over the ensemble of uniformly distributed molecules with orientations $x_{1}=-1, \ldots, x_{M}=1$. In both cases all of the molecules were initially in the ground state $|1\rangle$ and the target state was $|3\rangle$. The quality index $Q(\varepsilon)$ for this process in Eqn.(4) was maximized. The optimization

algorithm was a combination of tracking and a monotonic procedure [25], although we expect that similar results can be obtained with other monotonic algorithms [24]. In order to achieve physically relevant solutions it proved useful to slow down the convergence of the algorithm. This procedure is helpful to avoid large field intensities and also can be realized, in the case of the algorithm described in [26]. Atomic units are used in all of the calculations below. 


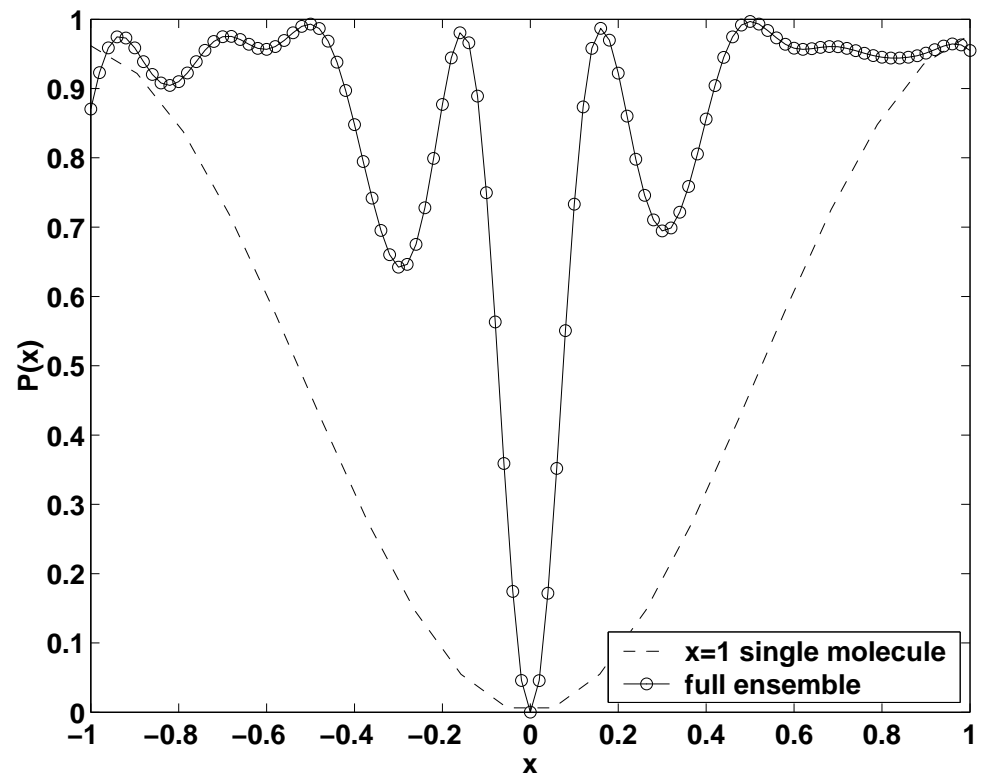

FIG. 1: The target yield $P(x)$ of $\mathrm{OH}$ vibrational excitation for different orientations $x=\cos (\theta)$. The yield $P(x)$ from a field optimized at $x=1$ produced the quality control index $Q(\varepsilon)=49 \%$. The associated field is given in Fig. 2. The yield $P(x)$ for full ensemble control of was required to optimize a sample of $M=31$ orientations uniformly distributed over the interval $[-1,1]$. The resultant quality index is $Q(\varepsilon)=85 \%$ and the associated optimal field is given in Fig. 3 .

Consider $\mathrm{OH}$ with unperturbed Hamiltonian $H_{0}=-\frac{\Delta}{2 m}+V$ where $m$ is the reduced mass of the system and $V$ is a Morse potential [24]:

$$
V(r)=D_{0}\left[e^{-\beta\left(r-r_{0}\right)}-1\right]^{2}-D_{0}, D_{0}=0.1994, \beta=1.189, r_{0}=1.821
$$

The dipole moment is taken as:

$$
\mu(r)=\mu_{0} r e^{-r / r^{*}}, \mu_{0}=3.088, r^{*}=0.6 .
$$

The target time is $T=2 \times 10^{5}$ a.u. $(\simeq 4.84 p s)$. Since the characteristic rotational time (i.e. the inverse of the rotational constant) for $\mathrm{OH}$ is $1.77 \mathrm{ps}$, the results presented here under the assumption of no reorientation during the pulse should represent a worst case control scenario as partial alignement of the molecule along the field would aid the control process.

The results for the case where all the molecules are oriented at $x=1$ is given in Figs. 1 and 2. Although the optimal control is performed at $x=1$, the same field in Fig. 2 was applied to the full ensemble of molecules over $-1 \leq x \leq 1$ producing the population 


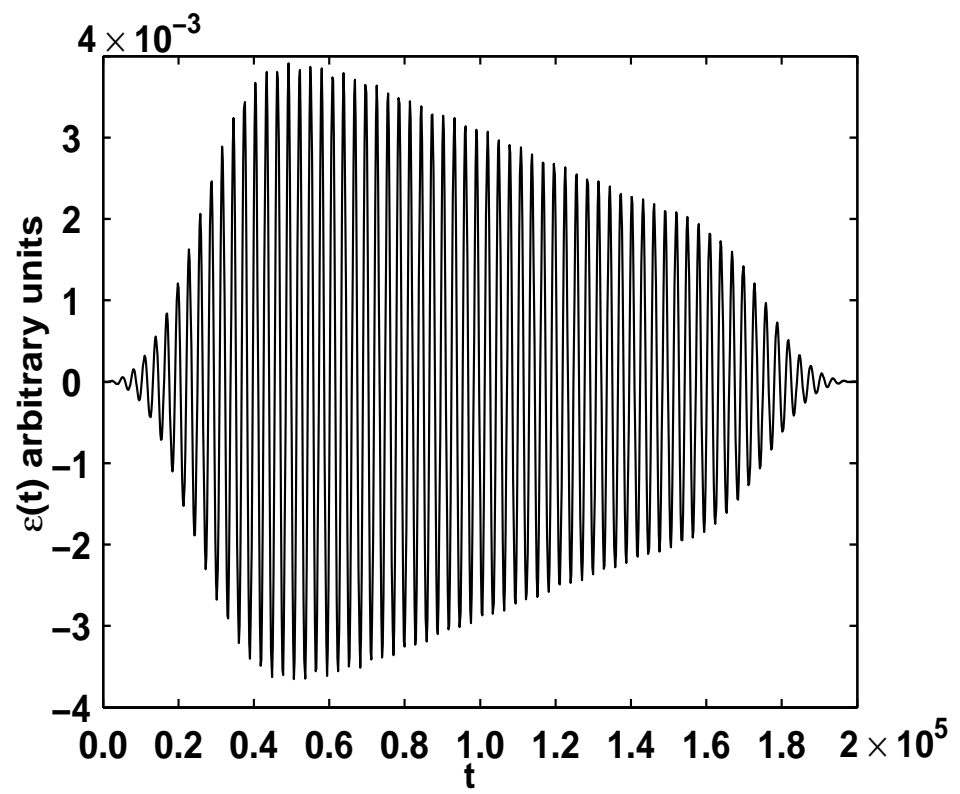

FIG. 2: The optimal field for $\mathrm{OH}$ that controls the $x=1$ oriented sample. The control performance is given in Fig. 1 for the field applied to molecules at any orientation. The field fluence is $\int_{0}^{T} \varepsilon(t)^{2} d t=0.64$

distribution $P(x)$ shown as a dashed line in Fig. 1. The latter distribution gradually goes to zero as $x \rightarrow 0$. The overall quality index of $Q(\varepsilon)=49 \%$ is rather good showing that approximately one half of the full ensemble of molecules can be controlled by the field determined at $x=1$. There is also evident reflection symmetry with respect to $x=0$ for control at different orientations. This behavior may be related to the system operating in the regime where the rotating wave approximation is valid. Some remnants of this behavior showed up in the other calculations, but the symmetry was not exploited and it is likely not generally valid for complex systems.

An ensemble consisting of $M=31$ uniformly sampled orientations over the interval $[-1,1]$ was subjected to optimal control. The control outcome is given in Fig. 1 and the associated optimal field is shown in Fig. 3. The yield distribution $P(x)$ in Fig. 1 is much enhanced over that achieved with the field at $x=1$ applied over $-1 \leq x \leq 1$. As a result, the new quality index $Q(\varepsilon)$ shows that $85 \%$ of the ensemble has been controlled. Naturally, those ensemble members near $x=0$ are more difficult to control. The enhanced control is at the expense of increasing the field fluence upon comparison of Figs. 2 and 3; even higher quality control 
indexes can be attained by further relaxing the fluence cost. The field structure has changed upon comparison of Figs. 2 and 3, but a simple scaling analysis of the field intensities is insightful. Consider the point $x=0.14$ which has a yield of $P(0.14)=96 \%$ for the full ensemble control in Fig. 1. To achieve this same yield the field in Fig. 2 determined at $x=1$ would need to be scaled to a fluence of $0.64 \cdot(1 / 0.14)^{2}=32.67$, which is comparable to the value of 50.68 in Fig. 3. The optimal field in Fig. 3 operates effectively at all $x$ values, while a different scaling of the field in Fig. 2 would be needed for each $x$ value to get the same results. This example clearly confirms the Theorem in Section II, and $Q$ is only kept from approaching $100 \%$ by constraints placed on the field fluence.

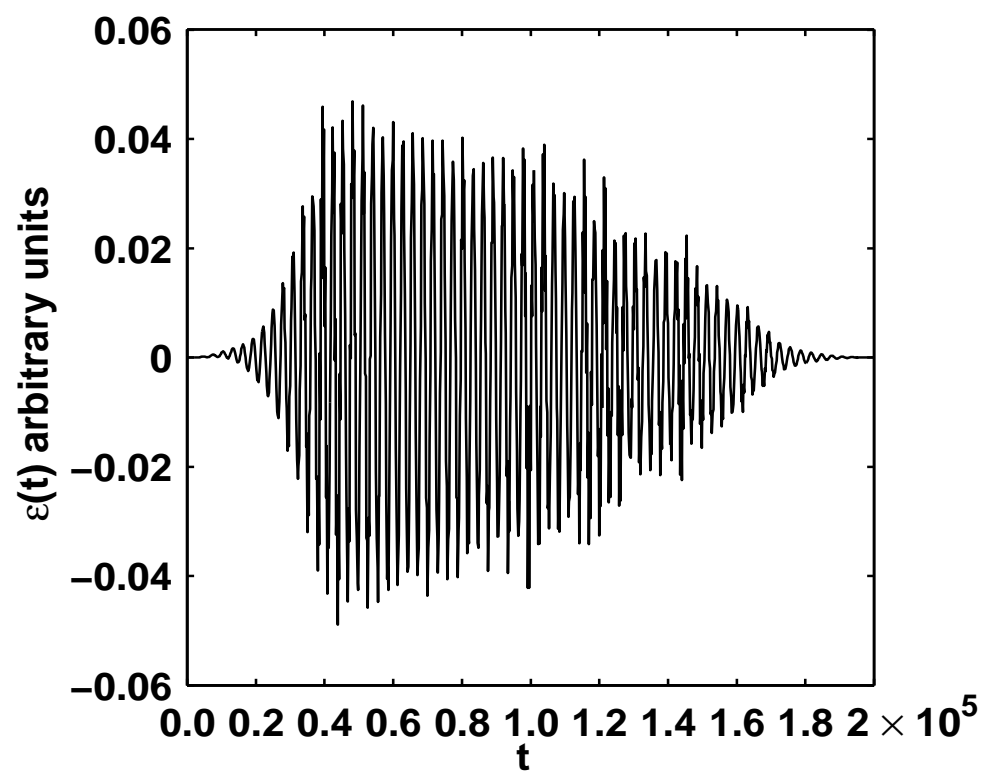

FIG. 3: The optimized field for $\mathrm{OH}$ that controls the full ensemble of molecules sampled at $M=31$ orientations. The performance of the field is given in Fig. 1. The field fluence is $\int_{0}^{T} \varepsilon(t)^{2} d t=50.68$.

The high quality overall yield of $85 \%$ for the full orientational ensemble in Fig. 1 arising from the field in Fig. 3 comes with distinct control mechanisms at the different $x$ values. This behavior is evident, for example, considering the controlled dynamics at $x=0.14,0.54$ and 1.0 shown in Figs. 4-6. In each case the yield $P(x)$ is approximately $96 \%$ but the dynamical paths taken by these three ensemble members are very different in Figs. 4-6. For example, in Fig. 4 at $x=0.14$ the target state is reached rather directly by the process $|1\rangle \rightarrow|3\rangle$ while at $x=1$ in Fig. 6 the state $|2\rangle$ plays a strong role and state the $|4\rangle$ is also involved. 


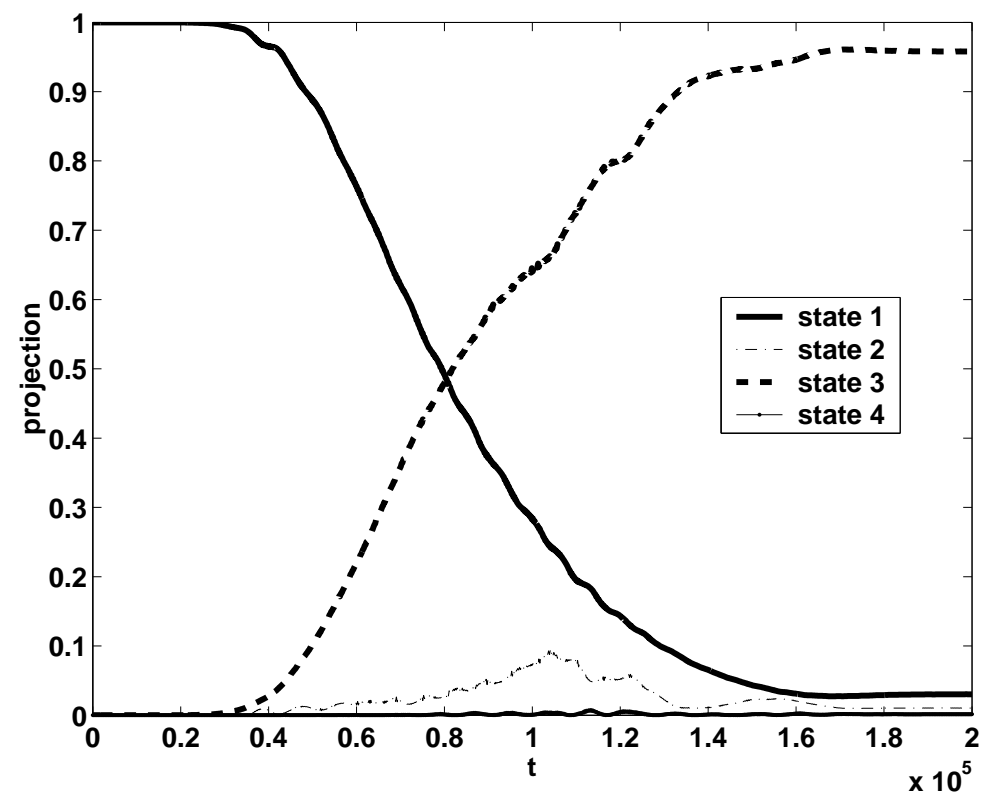

FIG. 4: Evolution of the populations of $\mathrm{OH}$ for $x=0.14$ with the optimal field controlling the full ensemble of randomly oriented molecules. This figure, upon comparison with Figs. 5 and 6, shows that the single field in Fig. 3 controlling the full ensemble induces distinctly different dynamics reaching the same target for differently oriented ensemble members.

Even more dramatic is the high degree of oscillatory structure in the population dynamics in amongst the four states in Fig. 5, and especially Fig. 6, which is absent in Fig. 4. Yet, all three ensemble members reach the same final target yield of $\sim 96 \%$ in state $|3\rangle$ at time $T$. This diversity of dynamical behavior has important implications for attempts at extracting and understanding the control mechanisms in the commonly occurring circumstance of manipulating randomly oriented ensembles of molecules. In particular, it is evident that mechanisms will have to be understood in a ensemble averaged sense.

\section{CONCLUSION}

This paper addresses the feasibility of controlling all members of an ensemble of randomly oriented molecules subjected to a single ultra-fast laser control pulse. Naturally, those molecules whose dipole is closer to being orthogonal to the applied field are more difficult to control. Except for the null set of molecules, whose dipole is exactly perpen- 


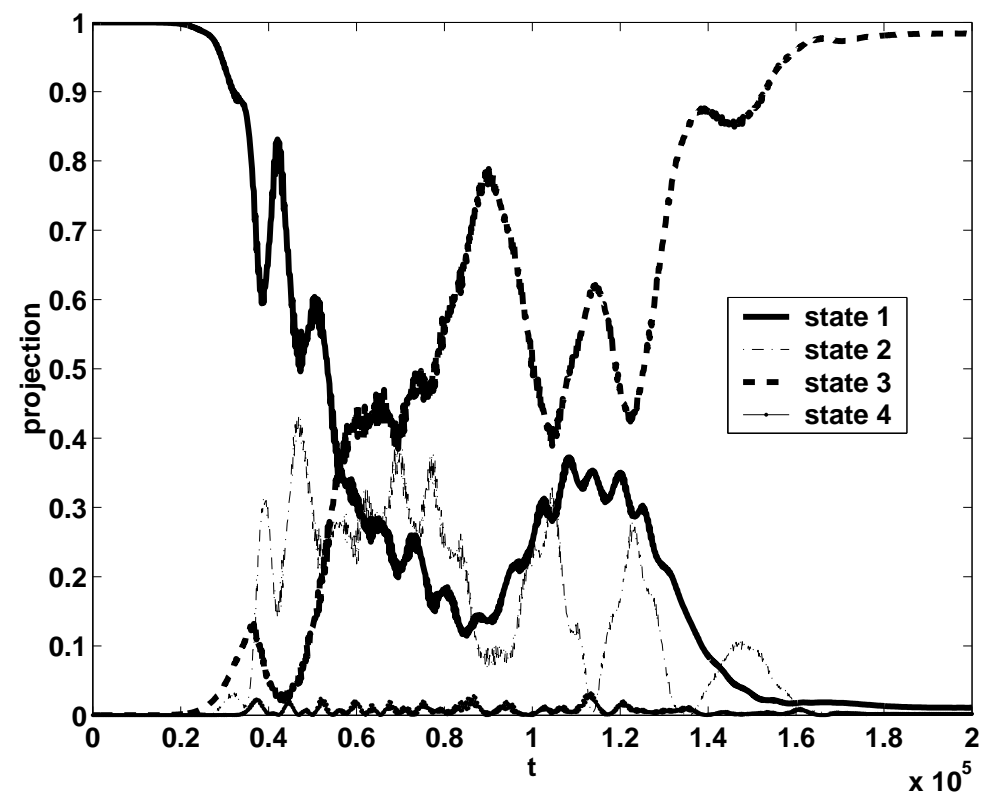

FIG. 5: Evolution of the populations of $\mathrm{OH}$ for $x=0.54$ (see Figure 4).

dicular to the field, all ensemble members can in principle be controlled by a single field as established by the Theorem proved in this paper. Numerical optimal control simulations verified this behavior to a practical degree. It is reasonable to also anticipate that some partial re-alignment as well as dynamic polarization will occur to likely beneficially ease the management of the more difficult to control fraction of the ensemble aligned initially perpendicular to the field. This situation could also be aided by the introduction of multi-polarization control fields [27].

In the laboratory, the closed loop adaptive control experiments will naturally seek out the best field to control all of the molecules in the medium taking into consideration orientational effects as well as laser beam spatial inhomogeneities[28]. This work indicates that a single field may be found which can give an excellent yield while simultaneously managing all of the ensemble members. This behavior is most encouraging for achieving maximum control in a bulk medium. However, understanding the control mechanism under these circumstances may present a complex matter for analysis. This work, and an analogous prior study considering spatial field effects [28], shows that each of the dynamically distinguishable members of the ensemble may follow a physically distinct pathway (i.e., mechanism) in reaching the same final target state. Thus, care will be needed to extract and understand 


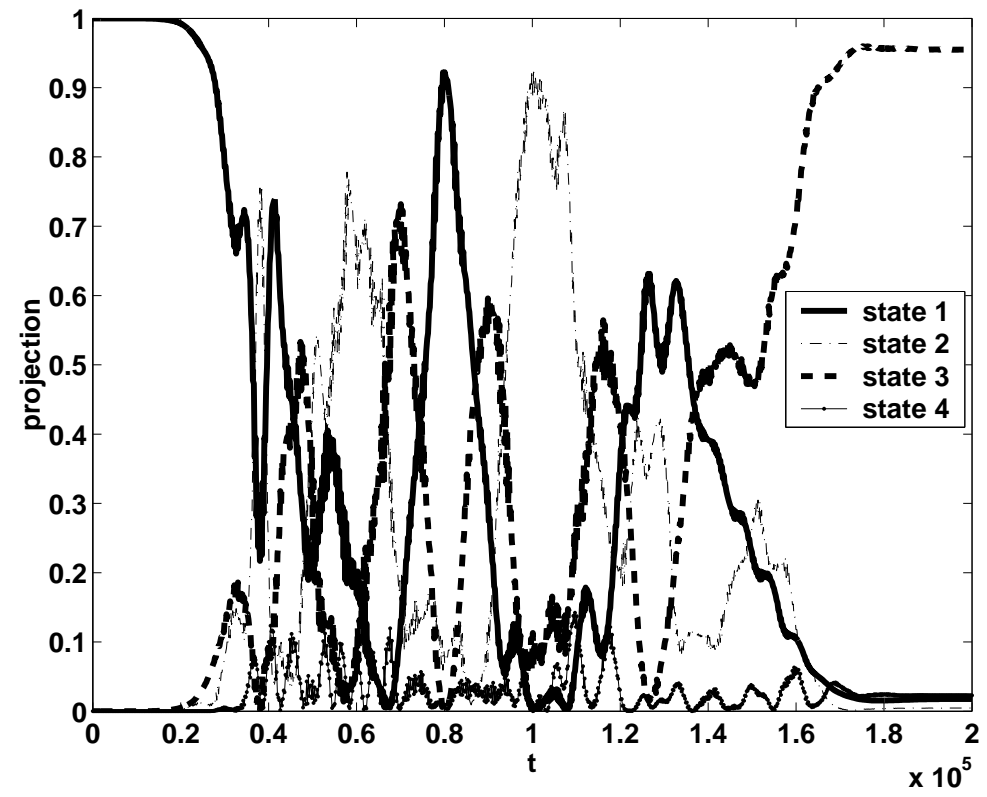

FIG. 6: Evolution of the populations of OH for $x=1.0$ (see Figure 4).

mechanistic insights from experiments carried out over randomly oriented ensembles as well as with laser beam spatial inhomogeneities. Just as the control process is an ensemble average, the control mechanism may also have to be viewed as having an ensemble average character.

\section{Acknowledgments}

H.R. acknowledges support from the National Science Foundation and an ARO-MURI grant.

\section{APPENDIX A: PROOF OF THE THEOREM}

Neither in the wave function nor in the density matrix representation does an overall phase play any role because only observable expectation values are considered. Thus we can safely suppose that both $H_{0}$ and $\mu$ are traceless. We will denote in the following by $\operatorname{diag}\left(d_{1}, \ldots, d_{P}\right)$ the matrix obtained by setting $d_{1}, \ldots, d_{P}$ on its diagonal. Here $d_{1}, \ldots, d_{P}$ may be complex numbers or square matrices.

Equation (1) is a collection of Schrödinger equations for $x_{k}=\cos \left(\theta_{k}\right), k=1, \ldots, M$ or, 
equivalently, a large system with block diagonal entries:

$$
i \hbar \frac{\partial}{\partial t}|\Psi(t)\rangle=\left(\mathcal{H}_{0}-\mathcal{B} \varepsilon(t)\right)|\Psi(t)\rangle
$$

where $\mathcal{H}_{0}=\operatorname{diag}\left(H_{0}, \ldots, H_{0}\right)$ is a $M N \times M N$ matrix constructed from $M$ replicas of $H_{0}$ and $\mathcal{B}=\operatorname{diag}\left(x_{1} \mu, \ldots, x_{M} \mu\right)$. Following a result from [29] (that can be used because of the compact setting), it suffices to prove that the Lie algebra $\mathcal{L}$ generated by the matrices $i \mathcal{H}_{0}$ and $i \mathcal{B}$ equals $\oplus_{k=1}^{M} s u(N)$.

We label by $e_{(a, b)}$ the $N \times N$ matrix whose entry at line $a$ and column $b$ is 1 and all others are zero and denote $Y_{(a, b)}=i\left(e_{(a, b)}+e_{(b, a)}\right), S_{(a, b)}=\left(e_{(a, b)}-e_{(b, a)}\right)$ and $D_{a}=$ $i\left(e_{(a, a)}-e_{(a+1, a+1)}\right)$. Note that $S_{(a, b)}, Y_{(a, b)},(a<b, a, b=1, N)$ and $D_{a}(a=1, \ldots, N-1)$ form a basis for $s u(N)$. We index by $\xi \in \Xi$ (of cardinality $K$ ) the entries $\xi=(a, b), a<b$ of the matrix $\mu$ that are non-zero: $\mu_{\xi=(a, b)} \neq 0$ and denote by $\xi^{\dagger}$ the pair $(b, a)$.

Consider now $a d_{i \mathcal{H}_{0}}^{\ell} i \mathcal{B}, \ell=1, \ldots, 2 K$ where $a d_{X}^{\ell} Y=[X, \ldots,[X, Y] \ldots]$ are the commutators taken $\ell$ times. A straightforward computation shows that $a d_{i \mathcal{H}_{0}}^{\ell} i \mathcal{B}$ is the block diagonal matrix $\operatorname{diag}\left(x_{1} a d_{i H_{0}}^{\ell} i \mu, \ldots, x_{K} a d_{i H_{0}}^{\ell} i \mu\right)$. Since $H_{0}$ is diagonal, $a d_{H_{0}}^{\ell} \mu=\left(\omega_{a b}^{\ell} \mu_{a b}\right)_{a, b=1, \ldots N}$. One can therefore explicitly list all commutators $a d_{i \mathcal{H}_{0}}^{\ell} i \mathcal{B}, \ell=1, \ldots, 2 K$ and compute their coefficients in the basis $\left\{\operatorname{diag}\left(x_{1} e_{\xi}, \ldots, x_{M} e_{\xi}\right), \operatorname{diag}\left(x_{1} e_{\xi^{\dagger}}, \ldots, x_{M} e_{\xi^{\dagger}}\right) ; \xi \in \Xi\right\}$. For instance, the coefficient of the $\operatorname{diag}\left(x_{1} e_{\xi}, \ldots, x_{M} e_{\xi}\right)$, in the development of $a d_{i \mathcal{H}_{0}}^{\ell} i \mathcal{B}$ is $i^{\ell+1}\left(\omega_{\xi}\right)^{\ell} \mu_{\xi}$ and that of $\operatorname{diag}\left(x_{1} e_{\xi^{\dagger}}, \ldots, x_{M} e_{\xi^{\dagger}}\right)$ is $i^{\ell+1}(-1)^{\ell}\left(\omega_{\xi}\right)^{\ell} \mu_{\xi}^{*}$. These coefficients can be put into a $2 K \times 2 K$ matrix

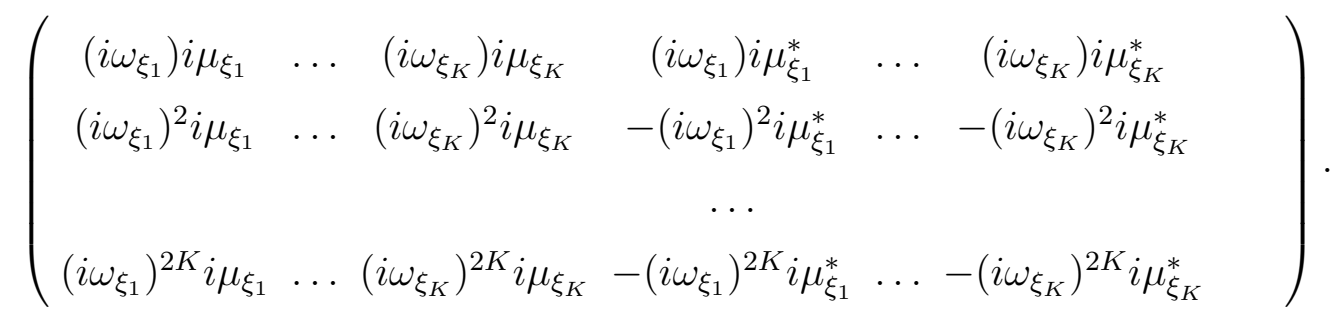

The determinant of this matrix can be computed explicitly. Although straightforward, this computation is cumbersome and we will not reproduce it here (see also [18]). It suffices to say that the determinant is zero only when $\left|\omega_{\xi}\right|=\left|\omega_{\xi^{\prime}}\right|$ for some $\xi \neq \xi^{\prime}$. Since this is prevented by hypothesis, the determinant is non-null which implies that $a d_{i \mathcal{H}_{0}}^{\ell} i \mathcal{B}, \ell=1, \ldots, 2 K$ are independent. Since all such matrices live in a $2 K$-dimensional vector space spanned by $\left\{\operatorname{diag}\left(x_{1} S_{\xi}, \ldots, x_{M} S_{\xi}\right), \operatorname{diag}\left(x_{1} Y_{\xi}, \ldots, x_{M} Y_{\xi}\right) ; \xi \in \Xi\right\}$ then they generate this space. We have therefore obtained that $\mathcal{S}=\operatorname{span}\left\{\operatorname{diag}\left(x_{1} S_{\xi}, \ldots, x_{M} S_{\xi}\right), \operatorname{diag}\left(x_{1} Y_{\xi}, \ldots, x_{M} Y_{\xi}\right) ; \xi \in \Xi\right\} \subset \mathcal{L}$. 
We now use the commutators of members of $\mathcal{S}$ to prove that additional matrices belong to the Lie algebra $\mathcal{L}$. This builds on the standard commutation relations among

$$
\begin{aligned}
& \text { For } a \neq b \neq c \neq a:\left[S_{(a, b)}, S_{(b, c)}\right]=S_{(a, c)},\left[S_{(a, b)}, Y_{(b, c)}\right]=Y_{(a, c)} \\
& {\left[D_{a}, S_{(a, b)}\right]=Y_{(a, b)},\left[D_{a}, Y_{(a, b)}\right]=S_{(b, a)},\left[S_{(a, a+1)}, Y_{(a, a+1)}\right]=D_{a}}
\end{aligned}
$$

Consider a path $i_{1}, \ldots, i_{L}$ in the graph $G$ (vertices are not necessarily unique). Recall that, since at least one individual system is controllable, the graph $G$ is connected [16] ; thus, a path exists between any two vertices.

We have seen that any $\operatorname{diag}\left(x_{1} S_{\left(i_{\ell}, i_{\ell+1}\right)}, \ldots, x_{M} S_{\left(i_{\ell}, i_{\ell+1}\right)}\right)$ is in $\mathcal{L}$. By iterating the commutators of these matrices as in Eqn. (A3) one obtains that $\operatorname{diag}\left(\left(x_{1}\right)^{L} S_{\left(i_{1}, i_{L}\right)}, \ldots,\left(x_{M}\right)^{L} S_{\left(i_{1}, i_{L}\right)}\right)$ is in $\mathcal{L}$ too. Since for any path $i_{1}, \ldots, i_{L}$ in $G$ the path $i_{1}, \ldots, i_{L}, i_{L-1}, i_{L}$ of length $L+2$ is also in $G$ it follows that $\operatorname{diag}\left(\left(x_{1}\right)^{L+2 j} S_{\left(i_{1}, i_{L}\right)}, \ldots,\left(x_{M}\right)^{L+2 j} S_{\left(i_{1}, i_{L}\right)}\right)$ is in $\mathcal{L}$ for any $j \geq 0$. As before, we select vectors for $j=0, \ldots, M$ and notice that, in the basis $\left(S_{\left(i_{1}, i_{L}\right)}, 0, \ldots, 0\right), \ldots$, $\left(0, \ldots, S_{\left(i_{1}, i_{L}\right)}, 0, \ldots, 0\right), \ldots,\left(0, \ldots, S_{\left(i_{1}, i_{L}\right)}\right)$ the coefficients form a Vandermonde type matrix $\left(\left(x_{k}\right)^{L+2 j}\right)_{k, j=1, \ldots K}$. Then the vectors are independent as soon as $\left|x_{k}\right| \neq\left|x_{k^{\prime}}\right|$ for $k \neq k^{\prime}$ which implies that

$$
\left(S_{\left(i_{1}, i_{L}\right)}, 0, \ldots, 0\right), \ldots,\left(0, \ldots, S_{\left(i_{1}, i_{L}\right)}, 0, \ldots, 0\right), \ldots,\left(0, \ldots, S_{\left(i_{1}, i_{L}\right)}\right) \in \mathcal{L}
$$

The same can be done for $\left(0, \ldots, Y_{(a, b)}, 0, \ldots, 0\right)$, and from here by standard commutation relations, all other remaining elements $\left(0, \ldots, D_{a}, 0, \ldots, 0\right)$ are recovered. Thus, $\mathcal{L}=\oplus_{k=1}^{M} s u(N)$.

Further care is needed when $\left|x_{k}\right|$ repeats in the list of orientation parameters (i.e. $x_{k^{\prime}}=$ $-x_{k}$ for some $\left.k, k^{\prime}\right)$. In this situation, as soon as one cycle of odd length $q$ exists in the graph, all elements $\operatorname{diag}\left(\left(x_{1}\right)^{L^{\prime}+j q} S_{\left(i_{1}, i_{L}\right)}, \ldots,\left(x_{M}\right)^{L^{\prime}+j q} S_{\left(i_{1}, i_{L}\right)}\right)$ for $j \geq 0$ can also be generated for some $L^{\prime}$ large enough. By the same arguments above one obtains again that all basis elements of the form $\left(0, \ldots, S_{\left(i_{1}, i_{L}\right)}, 0, \ldots, 0\right)$ belong to $\mathcal{L}$ which ends the proof.

[1] I. Walmsley and H. Rabitz, Physics Today 56, 43 (2003).

[2] R. Levis and H. Rabitz, J. Phys. Chem. A 106, 6427 (2002).

[3] T. Brixner, N. Damrauer, and G. Gerber, Adv. At., Mol., Opt. Phys. 46, 1 (2001).

[4] C. Daniel, J. Full, L. Gonzalez, C. Lupulescu, J. Manz, A. Merli, S. Vajda, and L. Woste, Science 299, 536 (2003). 
[5] R. Bartels, S. Backus, E. Zeek, L. Misoguti, G. Vdovin, I. Christov, M. Murnane, and H. Kapteyn, Nature 406, 164 (2000).

[6] J. Kunde, B. Baumann, S. Arlt, F. Morier-Genoud, U. Siegner, and U. Keller, Appl. Phys. Lett. 77, 924 (2000).

[7] J. Herek, W. Wohlleben, R. Cogdell, D. Zeidler, and M. Motzkus, Nature 417, 533 (2002).

[8] T. Weinacht, J. White, and P. Bucksbaum, The Journal of Physical Chemistry A 103, 10166 (1999).

[9] R.S. Judson and H. Rabitz, Phys. Rev. Lett (1992).

[10] M. Leibscher, I. S. Averbukh, and H. Rabitz, Phys. Rev. Lett. 90, 213001 (2003).

[11] M. Leibscher and I. S. Averbukh, Phys. Rev. A. 65, 053816 (2002).

[12] C.Z. Bisgaard, M.D. Poulsen, E. Péronne, S.S. Viftrup, and H. Stapelfeldt, Phys. Rev. Lett. 92, 173004 (2004).

[13] B. Li, G. Turinici, V. Ramakhrishna, and H. Rabitz, Journal of Physical Chemistry B 106, 8125 (2002).

[14] G. Turinici, V. Ramakhrishna, B. Li, and H. Rabitz, Journal of Physics A: Mathematical and General 37, 273 (2003).

[15] G. Turinici and H. Rabitz, Chem. Phys. 267, 1 (2001).

[16] G. Turinici and H. Rabitz, J. Phys.A. 36, 2565 (2003).

[17] S. G. Schirmer, H. Fu, and A.I. Solomon, Phys. Rev. A 63, 063410 (2001).

[18] C. Altafini, J.Math.Phys. 43, 2051 (2002).

[19] V. Ramakrishna, M.V. Salapaka, M. Dahleh, H. Rabitz, and A. Peirce, Phys. Rev. A 51 (2), 960 (1995).

[20] T. Kailath, Linear Systems (Prentice Hall, 1980).

[21] J. W. Polderman and J. C. Willems, Introduction to mathematical systems theory, vol. 26 of Texts in Applied Mathematics (Springer-Verlag, New York, 1998), ISBN 0-387-98266-3, a behavioral approach.

[22] M. Tucsnak and G. Weiss, SIAM J. Control Optim. 38, 1408 (2000), ISSN 1095-7138.

[23] H. Fu, S. Schirmer, and A.I. Solomon, J. Phys. A. 34 (2001).

[24] W. Zhu and H. Rabitz, J. Chem. Phys. 109, 385 (1998).

[25] M. Mirrahimi, G. Turinici, and P. Rouchon, in preparation (2004).

[26] Y. Maday and G. Turinici, J. Chem. Phys. 118 (2003). 
[27] T. Brixner, G. Krampert, T. Pfeifer, R. Selle, G. Gerber, M. Wollenhaupt, O. Graefe, C. Horn, D. Liese, and T. Baumert, Phys. Rev. Lett. 92, 208301 (2004).

[28] K. Sundermann, H. Rabitz, and R. de Vivie-Riedle, Phys. Rev. A 62, 013409 (2000).

[29] V. Jurdjevic and H. Sussmann, Journal of Differential Equations 12, 313 (1972). 